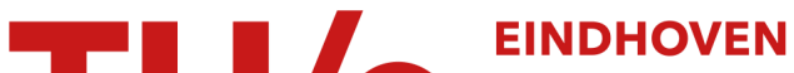 \\ UNIVERSITY OF \\ TECHNOLOGY
}

\section{Low-frequency noise in polysilicon emitter bipolar transistors}

Citation for published version (APA):

Markus, H. A. W., \& Kleinpenning, T. G. M. (1995). Low-frequency noise in polysilicon emitter bipolar transistors. IEEE Transactions on Electron Devices, 42, 720-727. https://doi.org/10.1109/16.372077

DOI:

10.1109/16.372077

Document status and date:

Published: 01/01/1995

\section{Document Version:}

Publisher's PDF, also known as Version of Record (includes final page, issue and volume numbers)

\section{Please check the document version of this publication:}

- A submitted manuscript is the version of the article upon submission and before peer-review. There can be important differences between the submitted version and the official published version of record. People interested in the research are advised to contact the author for the final version of the publication, or visit the $\mathrm{DOI}$ to the publisher's website.

- The final author version and the galley proof are versions of the publication after peer review.

- The final published version features the final layout of the paper including the volume, issue and page numbers.

Link to publication

\section{General rights}

Copyright and moral rights for the publications made accessible in the public portal are retained by the authors and/or other copyright owners and it is a condition of accessing publications that users recognise and abide by the legal requirements associated with these rights.

- Users may download and print one copy of any publication from the public portal for the purpose of private study or research.

- You may not further distribute the material or use it for any profit-making activity or commercial gain

- You may freely distribute the URL identifying the publication in the public portal.

If the publication is distributed under the terms of Article 25fa of the Dutch Copyright Act, indicated by the "Taverne" license above, please follow below link for the End User Agreement:

www.tue.nl/taverne

Take down policy

If you believe that this document breaches copyright please contact us at:

openaccess@tue.nl

providing details and we will investigate your claim. 


\title{
Low-Frequency Noise in Polysilicon Emitter Bipolar Transistors
}

\author{
H. A. W. Markus and T. G. M. Kleinpenning
}

\begin{abstract}
The low-frequency noise in polysilicon emitter bipolar transistors is investigated. Transistors with various geometries and various properties of the oxide layer at the monosilicon polysilicon interface are studied. The main $1 / f$ noise source proved to be located in the oxide layer. This source causes both $1 / f$ noise in the base current $S_{I_{b}}$ and $1 / f$ noise in the emitter series resistance $S_{r_{e}}$. The magnitude of the $1 / f$ noise source depends on the properties of the oxide layer. The $1 / f$ noise is ascribed to barrier height fluctuations of the oxide layer resulting in transparency fluctuations for both minority and majority carriers in the emitter, giving rise to $S_{I_{b}}$ and $S_{r_{e}}$, respectively. It is also shown that a low transparency of the oxide layer also reduces the contribution of mobility fluctuations to $S_{I_{b}}$
\end{abstract}

\section{INTRODUCTION}

$\mathrm{N}$ most recent papers the $1 / f$ noise in bipolar junction transistors (BJT) with a conventional emitter is mainly discussed in terms of mobility fluctuations. The $1 / f$ noise in BJTs has been interpreted in terms of two $1 / f$ noise current generators: $S_{I_{b}}$ between emitter and base, and $S_{I_{c}}$ between emitter and collector. In practical cases the basecollector junction is reverse biased, so that the leakage basecollector current is small and its noise negligible. Recently, Kleinpenning [1] derived formulas for the low-frequency noise in BJTs incorporating the noise sources present in the parasitic series resistances.

Several authors have studied the low-frequency noise in polysilicon emitter BJTs [1]-[6]. The low-frequency noise always consists of white shot noise and $1 / f$ noise. Sometimes burst noise is observed. Kleinpenning [1] found that the $1 / f$ noise was located in the base current $S_{I_{b}}$ and that its spectral density was proportional to the base current $I_{B}$. He interpreted the $1 / f$ noise in terms of mobility fluctuations. Pong-Fei Lu [2] also found the $1 / f$ noise to be located in the base current, but he found $S_{I_{b}} \sim I_{B}^{2}$. Pawlikiewicz et al. [3] found $S_{I_{b}} \sim I_{B}^{2}$ at higher currents and $S_{I_{b}} \sim I_{B}$ at lower currents. SiabiShahrivar et al. [4] and Mounib et al. [5] showed that the $1 / f$ noise is strongly related to the surface treatment prior to polysilicon deposition. Wai Shing Lau et al. [6] found $S_{I_{b}} \sim I_{B}^{2}$. They suggested that transparency fluctuations of the oxide layer, present at the monosilicon-polysilicon interface, or two-step tunnelling via traps in the oxide is responsible for the observed $1 / f$ noise. However, they did not give a detailed quantitative analysis.

Manuscript received May 5, 1994; revised November 29, 1994. The review of this paper was arranged by Associate Editor J. R. Hauser.

The authors are with the Eindhoven University of Technology, Department of Electrical Engineering, $5600 \mathrm{MB}$ Eindhoven, The Netherlands.

IEEE Log Number 9409065.
This paper will deal with the location of the main $1 / f$ noise source in polysilicon emitter BJTs with an oxide layer at the monosilicon-polysilicon interface. A model based on transparency fluctuations of the oxide layer, that can describe this noise source, will be presented. By means of this model, it will be shown that the contribution of the mobility fluctuations to $S_{I_{b}}$ is overrun by transparency fluctuations.

\section{EXPERIMENTAL RESULTS}

\section{A. Devices}

The transistors are made by Philips Electronics. Transistors from 7 wafers were studied. On each wafer a set of transistors with various geometries is present. The length of the emitter (perpendicular to the base current) is in the range of $0.8 \mu \mathrm{m}<\mathrm{L}<100 \mu \mathrm{m}$, and its width (parallel to the base current) in the range of $0.3 \mu \mathrm{m}<\mathrm{W}<100 \mu \mathrm{m}$. For transistors from four wafers a HF dip is used to produce a native oxide layer at the polysilicon-monosilicon interface with an estimated thickness in the range of $10-15 \AA$. This native layer was removed from the other three wafers by a $\mathrm{HF} / \mathrm{H}_{2} \mathrm{O}$ vapor etch in a cluster tool and, subsequently, a new layer was thermally grown with different oxidation times. The average thicknesses of the oxide layers given by the manufacturer are $3.8 \AA, 7.5 \AA$ and $8 \AA$, respectively. The emitter drive-in is a furnace anneal at $1173 \mathrm{~K}$ during 30 minutes. The top-doping of the emitter of all transistors is estimated to be $10^{20} \mathrm{~cm}^{-3}$. The junction depth is in the range of 57-71 nm. Only n-p-n type transistors made with this process were available.

\section{B. I-V Characteristics}

Fig. 1 shows a typical I-V curve of the base and collector current versus $V_{B E}$ at $V_{C B}=3 \mathrm{~V}$. The ideality factor for the base and collector current is 1 . The current gain $\beta$ collapses for high currents, which is ascribed to high injection in the base. Current crowding is negligible in the studied current range.

With the help of white noise measurements in commoncollector configuration [1], we found the values of $4 k T\left(r_{e}+\right.$ $\left.r_{b}\right)$. From the deviation of the exponential behaviour of the I-V characteristics, we found $I_{E} r_{e}+I_{b} r_{b}$. Hence, the values of the emitter series resistance $r_{e}$ and the internal base resistance $r_{b}$ can be determined. The values found for $r_{b}$ agree well with the calculated values of the sum of the base sheet resistance given by the manufacturer, and the resistance between base contacts and emitter edge. At high currents the base sheet resistance can become bias dependent due to current crowding and high 


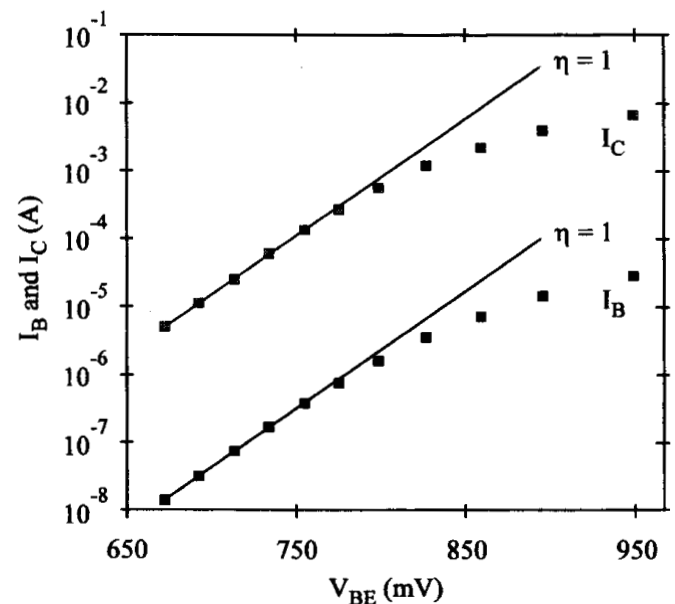

Fig. 1. The I-V curves of a polysilicon emitter bipolar transistor with an emitter area of $0.3 \times 48 \mu \mathrm{m}^{2}$. The grown oxide-layer thickness is $8 \AA$ $V_{C B}=3 \mathrm{~V}$

injection. However, an important part of the base resistance is formed by the resistance between the base contacts and the emitter edge. As a result we expect the base resistance to be only weakly bias dependent in the studied current range. Our experimentally obtained $\mathrm{I}-\mathrm{V}$ characteristic can be interpreted satisfactorily with a constant base resistance. The value of $r_{e}$ depends on the emitter geometry and the oxide thickness, so it is obvious that the tunnel resistance of the oxide layer is an important part of the total emitter resistance. The experimentally observed product of the emitter series resistance times the emitter area, $r_{e} A_{E}$, is in the range of $40 \Omega \mu \mathrm{m}^{2}-160 \Omega \mu \mathrm{m}^{2}$. In the studied current range the voltage across the oxide layer $(<100 \mathrm{mV}$ in the studied current range) was much smaller than the effective barrier height $(0.6 \mathrm{~V})$, so the part of the emitter series resistance due to tunneling is assumed to be ohmic.

\section{Noise Measurements}

We made the noise measurements by putting the transistor in a circuit as shown in Fig. 2. The internal series resistances are given by $r_{e}, r_{b}$, and $r_{c}$ respectively. Three external metal film resistors $R_{B}, R_{C}$, and $R_{E}$ can be inserted which only have Nyquist noise ( $4 k T R)$. According to Kleinpenning [1], the $1 / f$ voltage noise across $R_{E}$ and $R_{C}$ can be written as

$$
\begin{aligned}
\left(Z / R_{E}\right)^{2} S_{V_{E}}= & {\left[r_{\pi}-\beta\left(r_{b}+R_{B}\right)\right]^{2} S_{I_{b}} } \\
& +\left[r_{\pi}+r_{b}+R_{B}\right]^{2} S_{I_{c}} \\
& +(\beta+1)^{2}\left[I_{B}^{2} S_{r_{b}}+I_{E}^{2} S_{r_{e}}\right] \\
\left(Z / R_{C}\right)^{2} S_{V_{C}}= & \beta^{2}\left[r_{b}+R_{B}+r_{e}+R_{E}\right]^{2} S_{I_{b}} \\
& +\left[r_{\pi}+r_{b}+R_{B}+r_{e}+R_{E}\right]^{2} S_{I_{c}} \\
& +\beta^{2}\left[I_{B}^{2} S_{r_{b}}+I_{E}^{2} S_{r_{e}}\right]
\end{aligned}
$$

with

$$
\begin{aligned}
Z & =r_{b}+R_{B}+r_{\pi}+(\beta+1)\left(r_{e}+R_{E}\right) \\
r_{\pi} & =d V_{b e} / d I_{B} \\
\beta & =d I_{C} / d I_{B}=g_{m c} \cdot r_{\pi} .
\end{aligned}
$$

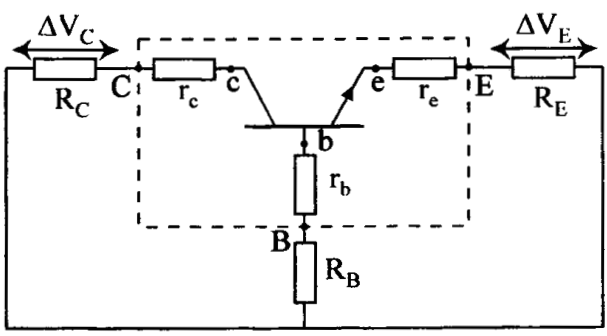

Fig. 2. AC noise measurement circuit.

Here, $g_{m c}$ represents the collector transconductance, $\beta$ the current amplification factor, and $r_{\pi}$ the junction differential resistance of the intrinsic transistor. $V_{b e}$ is the voltage drop across the emitter-base junction. $S_{I_{b}}$ and $S_{I_{c}}$ represent the $1 / f$ current noise spectral density at constant $V_{b e}$ in the base and collector currents, respectively. The series resistances $r_{e}$ and $r_{b}$ can have $1 / f$ resistance noise. Their spectral densities are represented by $S_{r_{e}}$ and $S_{r_{b}}$, respectively. The Early effect was ignored in the derivation of (1) and (2). This is permissible since $I_{C}$ was found to be almost independent of $V_{C B}$ at $V_{C B}>1 \mathrm{~V}$.

In order to locate and characterize the $1 / f$ noise sources, we carried out our measurements in both a common-emitter (C-E) configuration and a common-collector $(\mathrm{C}-\mathrm{C})$ configuration at various biasing conditions.

In the C-C configuration we took $R_{E} \gg r_{e}, r_{b}, r_{\pi}, R_{B}$, so that $Z / R_{E}=\beta+1$, and $R_{C}=0$. Thus, (1) simplifies to

$$
\begin{aligned}
g_{m e}^{2} S_{V_{E}}= & {\left[1-g_{m c}\left(r_{b}+R_{B}\right)\right]^{2} S_{I_{b}}+\left[1+\left(r_{b}+R_{B}\right) / r_{\pi}\right]^{2} } \\
& \cdot S_{I_{c}}+g_{m e}^{2}\left[I_{B}^{2} S_{r_{b}}+I_{E}^{2} S_{r_{e}}\right] .
\end{aligned}
$$

Here, $g_{m e}=(\beta+1) / r_{\pi}=d I_{E} / d V_{b e}$ is the emitter transconductance of the intrinsic transistor.

In the C-E configuration we put $R_{E}=0, R_{B} \gg$ $r_{b}, r_{\pi},(\beta+1) r_{e}$, and $R_{C}=100 \Omega$, so that $Z=R_{B}$. Then, (2) reduces to

$$
S_{V_{C}} / R_{C}^{2}=\beta^{2} S_{I_{b}}+S_{I_{c}}+\left(\beta / R_{B}\right)^{2}\left[I_{B}^{2} S_{r_{b}}+I_{E}^{2} S_{r_{e}}\right] \text {. }
$$

The noise measurements were performed in the current ranges $0.1 \mu \mathrm{A} \leq I_{B} \leq 100 \mu \mathrm{A}$ and $10 \mu \mathrm{A} \leq I_{C} \leq 10 \mathrm{~mA}$. In these current ranges the input resistance $r_{\pi}=k T / q I_{B}$ varies from $250 \Omega$ to $250 \mathrm{k} \Omega$, the collector transconductance $g_{m c}=q I_{C} / k T$ from $4 \cdot 10^{-4} \Omega^{-1}$ to $0.4 \Omega^{-1}$, and the emitter transconductance $g_{m e}=g_{m c}+1 / r_{\pi}$ from $4 \cdot 10^{-4} \Omega^{-1}$ to $0.4 \Omega^{-1}$. These parameters, being the parameters of the transistor without series resistances, are different from those of the complete transistor that are defined by $d V_{B E} / d I_{B}, d I_{C} / d V_{B E}$ and $d I_{E} / d V_{B E}$, respectively, with $V_{B E}=V_{b e}+I_{B} r_{b}+I_{E} r_{e}$.

Spectral noise densities were measured from $1 \mathrm{~Hz}$ to 100 $\mathrm{kHz}$. All devices showed $1 / f$ noise. The $1 / f$ spectral density was observed over at least one decade of frequency, but depending on emitter geometry and current reaching up to 5 decades of frequency. Using (6) and (7), we can determine the location of the dominant noise sources. In (6) both $S_{I_{b}}$ and $S_{I_{c}}$ have a prefactor of 1 at low currents, where $g_{m c}\left(r_{b}+R_{B}\right) \ll 1$ and $\left(r_{b}+R_{B}\right) / r_{\pi} \ll 1$. However in (7) the contribution of $S_{I_{b}}$ only is amplified by a factor $\beta^{2}$. In (6) the noise contribution 


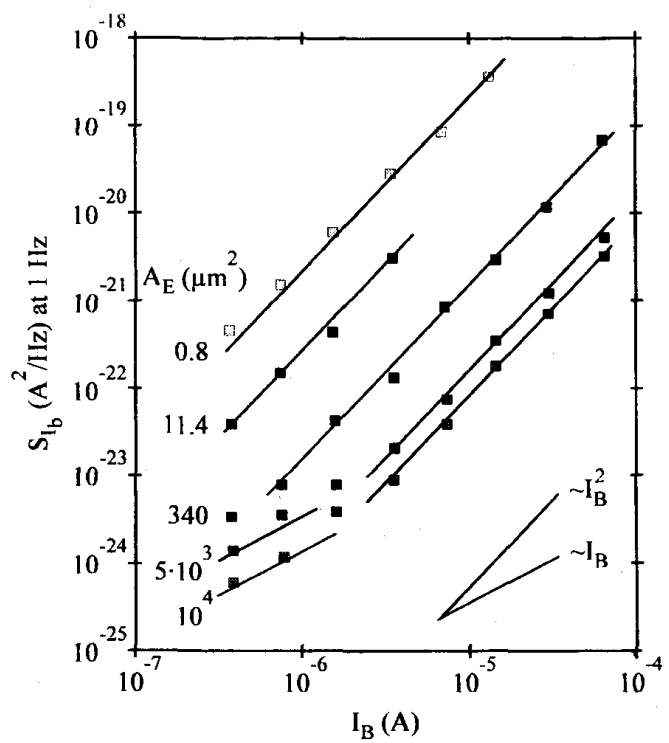

Fig. 3. $S_{I_{b}}$ versus $I_{B}$ for transistors with $7,5 \AA$ grown oxide layer and various emitter areas.

of the series resistances $S_{r_{b}}$ and $S_{r_{e}}$ is amplified by a factor $\beta^{2}$, whereas in (7) it is only amplified by $\left(\beta / R_{B}\right)^{2}$. Hence, by measuring both in the C-E and C-C configuration at various values of $V_{e b}$ and $R_{B}$, one can decide whether $S_{I_{b}}$ or $S_{I_{c}}$, or the contribution of the series resistances is dominant. Our measurements revealed that $S_{I_{b}}$ and $S_{r_{e}}$ are dominant.

In Fig. 3 some typical plots are presented of the spectral density of the base current $1 / f$ noise $S_{I_{b}}$ at $1 \mathrm{~Hz}$ versus $I_{B}$. The results presented were obtained from transistors with a grown oxide layer of $7.5 \AA$ and various emitter areas. One can observe that $S_{I_{b}} \sim I_{B}^{2}$. Only the transistors with a large emitter area show a deviation from this square law at low currents.

In Fig. 4 the relative noise $S_{I_{b}} / I_{B}^{2}$ from the same devices is plotted versus the emitter area $A_{E}$. One can observe that $S_{I_{b}} / I_{B}^{2} \sim A_{E}^{-1}$.

In Fig. 5 the relative noise $S_{I_{b}} / I_{B}^{2}$ from the transistors with grown oxide layers and an emitter area of $8.8 \times 48 \mu \mathrm{m}^{2}$ is plotted versus the oxide thickness. One can observe a clear influence of the oxide layer thickness on the $1 / f$ base current noise.

The contribution of resistance fluctuations of the parasitic emitter and base resistance can be measured in the C-C configuration. By taking $R_{B}=0$ we can approximate expression (6) for high currents, where $I_{C} \gg k T / q r_{b}$, by

$$
S_{V_{E}} \approx r_{b}^{2} S_{I_{b}}+I_{E}^{2} S_{r_{e}}+I_{B}^{2} S_{r_{b}}
$$

For transistors with a small emitter area, the $S_{V_{E}}$ measured was up to 3 orders of magnitude higher than $r_{b}^{2} S_{I_{b}}$. Here, the values of $S_{I_{b}}$ were used that were measured in the C-E configuration. So, at high currents $S_{V_{E}}$ is determined either by base resistance fluctuations or by emitter resistance fluctuations. In Section IV we will discuss this in more detail.

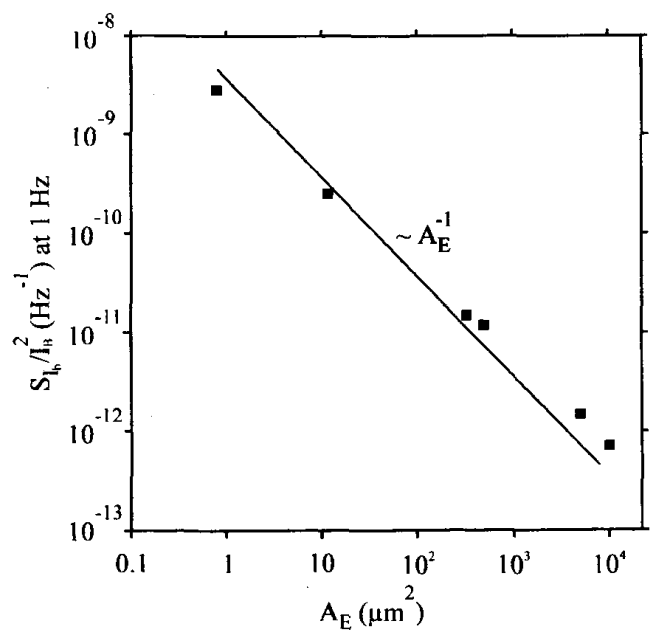

Fig. 4. $S_{I_{b}} / I_{B}^{2}$ versus $A_{E}$ of all transistors with $7,5 \AA$ grown oxide layer.

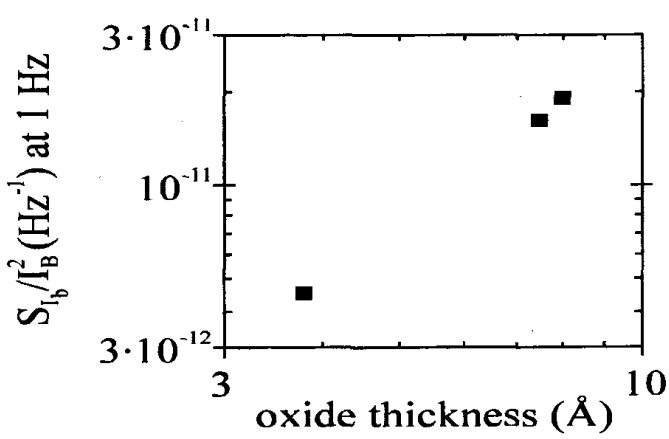

Fig. 5. $S_{I_{b}} / I_{B}^{2}$ versus the oxide thickness for transistors with grown oxide layers of $3.8 \AA, 7.5 \AA$ and $8 \AA$ and an emitter area of $8.8 \times 48 \mu \mathrm{m}^{2}$.

\section{THEORETICAL MODEL}

\section{A. General Remarks}

Suppose one finds $S_{I_{b}} \sim I_{B}^{k}$ experimentally. If the current density, $J_{B}$, is homogeneously distributed over the emitter area and the noise sources are also homogeneously distributed over the emitter area and are spatially uncorrelated, then one can derive the following spectral noise density of $J_{B}$ [7]:

$$
S_{J_{B}} \sim J_{B}^{k}=\left(I_{B} / A_{E}\right)^{k}
$$

which leads to

$$
S_{I_{B}} \sim A_{E} S_{J_{B}}=I_{B}^{k} \cdot A_{E}^{1-k} .
$$

We found $S_{I_{b}} \sim I_{B}^{2}$ experimentally (see Fig. 3). As a result (9) predicts $S_{I_{b}} / I_{B}^{2} \sim A_{E}^{-1}$ which agrees well with our experiments (see Fig. 4). This is a strong indication for homogeneously distributed $1 / f$ noise sources.

In conventional $\mathrm{p}-\mathrm{n}$ junctions the $1 / f$ noise can be interpreted in terms of mobility fluctuations. The mobility fluctuation model [8] predicts a linear relationship between $S_{I_{b}}$ and $I_{B}$. However, we found $S_{I_{b}} \sim I_{B}^{2}$. As a consequence, our results cannot be explained in terms of mobility fluctuations 
only. A new model for $S_{I_{b}} \sim I_{B}^{2}$ will be presented in Section III-B.

In Fig. 3 we observe deviations from the square law for the larger emitter areas at small currents. In this region $S_{I_{b}}$ seems to be proportional to $I_{B}$, so that mobility fluctuations might be the dominant noise source here. In Section III.D, a relation will be derived for the contribution of mobility fluctuations to $S_{I_{b}}$, taking into account the influence of the oxide layer at the polysilicon-monosilicon interface.

A linear relation between $S_{I_{b}}$ and $I_{B}$ can also be found when the ideal base current dominates the base current and the non-ideal base current (with ideality factor 2) dominates the $1 / f$ noise, provided that the $1 / f$ noise is proportional to the non-ideal base current squared. Such a quadratic dependence is not predicted by the mobility fluctuation model.

\section{B. Transparency Fluctuation Model}

One of the $1 / f$ noise model predicting $S_{I} \sim I^{2}$ is the tunnel diode model proposed by Kleinpenning [9], [10]. He assumed that the Nyquist noise of the insulator layer modulates the barrier height and hence the tunneling probability, or transparency, of carriers. He derived the following relation for the relative noise in the transparency, that is valid for a biasing voltage much lower than the effective barrier height.

$$
\frac{S_{t}}{t^{2}}=\frac{2 m q k T L^{2} \operatorname{Re}(Z)}{3 V_{o} \hbar^{2}} \text { with } \operatorname{Re}(Z)=\frac{\tan \delta}{2 \pi f C} .
$$

Here, $t$ is the transparency of the oxide layer, $m$ the effective mass of the carrier, $T$ the temperature, $L$ the thickness of the oxide layer, $V_{0}$ the barrier height, $C$ the capacitance of the oxide layer, $\operatorname{Re}(Z)$ the real part of the impedance of the layer, and $\tan \delta$ the loss factor of the oxide.

Fluctuations of the barrier height of the oxide at the polysilicon-monosilicon interface results in fluctuations of the transparency of the barrier for the holes injected in the emitter, and so in base current fluctuations.

The following calculation yields the relation between the relative noise in the transparency, given by (10), and the relative noise in the short circuit base current. In Fig. 6 a schematic plot is given of the emitter structure of a polysilicon emitter, where we can distinguish the monocrystalline layer, the oxide layer, the polysilicon layer, and a metal contact. The minority carrier concentration in the monosilicon and polysilicon layer is also depicted. Here, we neglect carrier recombination in the monocrystalline and polycrystalline layer. This is allowed provided that $W_{m}^{2} / 2 D_{m} \ll \tau_{m}$ and $W_{p}^{2} / 2 D_{p} \ll \tau_{p}$, with $\tau_{m}$ and $\tau_{p}$ being the hole lifetime in the monosilicon and polysilicon layer, respectively, and provided that $s_{o x p} \gg$ $W_{m} / \tau_{m}, s_{m} \gg W_{m} / \tau_{m}$, and $s_{m} \gg W_{p} / \tau_{p}$, with $s_{o x p}$ and $s_{m}$ being the hole recombination velocity of the oxide layer and the metal, respectively. Furthermore, we assume a uniform doping within the emitter. The current can then be calculated from the following equations

$$
\begin{array}{ll}
\text { monosilicon layer: } & I=-q A_{E} D_{m} d p(x) / d x \\
\text { polysilicon layer: } & I=-q A_{E} D_{p} d p(x) / d x \\
\text { metal contact: } & I=q A_{E} s_{m}\left[p\left(x_{3}\right)-p_{0_{p}}\right] .
\end{array}
$$

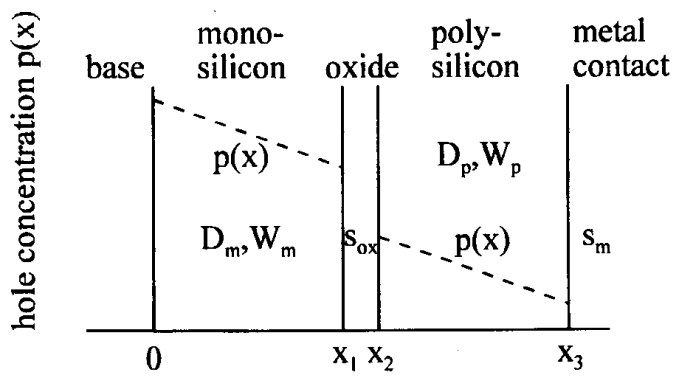

Fig. 6. Schematic picture of the polysilicon emitter with the hole concentration $p(x)$.

Here, $D_{m}$ is the diffusivity of holes in the monosilicon layer, $D_{p}$ the diffusivity of holes in the polysilicon layer, and $p_{0_{p}}$ the equilibrium hole concentration at $x=x_{3}$. The minority carrier tunneling current through the oxide barrier is given by the following relation [11]

$$
I=q A_{E} \sqrt{\frac{k T}{2 \pi m_{p}^{*}}} t_{p}\left[p\left(x_{2}\right)-p\left(x_{1}\right) e^{-q V / k T}\right] .
$$

Here, $m_{p}^{*}$ is the effective mass of holes in the oxide, $t_{p}$ is the tunneling probability or transparency for holes and $V$ the voltage across the barrier.

For intermediate emitter currents where $V \ll k T / q$, (14) can be approximated by

$$
I=q A_{E} s_{o x p}\left[p\left(x_{1}\right)-p\left(x_{2}\right)\right] .
$$

Here, $s_{o x p}$ is the oxide recombination velocity of holes given by

$$
s_{o x p}=\sqrt{\frac{k T}{2 \pi m_{p}^{*}}} t_{p} .
$$

Solving (11)-(13) and (15) for $I$ yields

$$
\begin{aligned}
I & =\frac{q A\left[p(0)-p_{0 p}\right]}{\frac{1}{s_{o x p}}+\frac{1}{s_{m}}+\frac{W_{m}}{D_{m}}+\frac{W_{p}}{D_{p}}} \text { with } \\
p(0) & =\frac{n_{i}^{2}}{N_{E}} e^{q V_{b e} / k T} .
\end{aligned}
$$

Here, $n_{i}$ is the intrinsic carrier concentration in the emitter, and $N_{E}$ the emitter dopant concentration.

Now, we can calculate the contribution of the fluctuations in the oxide recombination velocity to the current fluctuations in the base current. Since, $\Delta I_{b}=\left(d I / d s_{\text {ox } p}\right) \Delta s_{o x p}$, we find

$$
\begin{aligned}
\frac{S_{I_{b}}}{I_{B}^{2}} & =\left(\frac{d \ln I}{d \ln s_{o x p}}\right)^{2} \frac{S_{s_{o x p}}}{s_{o x p}^{2}} \\
& =\left[1+s_{o x p}\left(\frac{1}{s_{m}}+\frac{W_{m}}{D_{m}}+\frac{W_{p}}{D_{p}}\right)\right]^{-2} \frac{S_{s_{o x p}}}{s_{o x p}^{2}} .
\end{aligned}
$$

From (10) and (16) it follows that

$$
\frac{S_{s_{o x p}}}{s_{o x p}^{2}}=\frac{S_{t_{p}}}{t_{p}^{2}}=\frac{2 m_{p}^{*} q k T L^{2} \operatorname{Re}(Z)}{3 V_{0} \hbar^{2}} .
$$


Barrier height fluctuations of the oxide layer also modulate the tunneling probability, or transparency $t_{n}$, for the majority carriers and hence generates $1 / f$ noise in the emitter series resistance. For the barrier in the ohmic region, we have

$$
S_{r_{o x}} / r_{o x}^{2}=S_{t_{n}} / t_{n}^{2} \approx S_{r_{e}} / r_{e}^{2}
$$

provided that the emitter resistance $r_{e}$ is determined by the tunnel resistance $r_{o x}$.

\section{Two-Step Tunneling}

In Section III.B we ascribed the $1 / f$ noise to fluctuations in the barrier height of the oxide. Another explanation for the $1 / f$ noise generated in the oxide layer is proposed by Kumar et al. [12]. They propose that two-step tunneling via traps in the oxide is responsible for the $1 / f$ noise. They assume that the oxide contains electrically chargeable states which communicate with interface states by tunneling and have time constants dependent on the depth of the traps in oxide. Very specific requirements with respect to the trap distribution have to be met in order to obtain a $1 / f$ spectrum. The current dependence is $S_{I} \sim I^{2}$. Application of this theory also yields reasonable results. Both theories predict a different dependence on the oxide layer thickness. However, the range of oxide layer thicknesses of our transistors is too small to distinguish between both theories.

\section{Mobility Fluctuations}

In the appendix the contribution of mobility fluctuations to $S_{I_{b}}$ is calculated to be

$$
S_{I}(f)=\frac{\frac{\alpha_{m} q I}{D_{m} f} \ln \left[\frac{p(0)}{p\left(x_{1}\right)}\right]+\frac{\alpha_{p} q I}{D_{p} f} \ln \left[\frac{p\left(x_{2}\right)}{p\left(x_{3}\right)}\right]}{\left(\frac{W_{m}}{D_{m}}+\frac{W_{p}}{D_{p}}+\frac{1}{s_{o x}}+\frac{1}{s_{m}}\right)^{2}} .
$$

Here, $\alpha_{m}$ and $\alpha_{p}$ are the Hooge parameters of the monosilicon and polysilicon, respectively.

From the expressions (11)-(13) and (15), we can find the ratios $p(0) / p\left(x_{1}\right)$ and $p\left(x_{3}\right) / p\left(x_{2}\right)$ for $q V_{b e} \gg k T$ being

$$
\begin{aligned}
\frac{p(0)}{p\left(x_{1}\right)}= & 1+\frac{W_{m}}{D_{m}}\left(\frac{1}{s_{o x}}+\frac{1}{s_{m}}+\frac{W_{p}}{D_{p}}\right)^{-1} \text { and } \\
& \frac{p\left(x_{3}\right)}{p\left(x_{2}\right)} \approx 1+\frac{s_{m} W_{p}}{D_{p}} .
\end{aligned}
$$

\section{DISCUSSION OF NOISE RESUlTS}

With the aid of (21) and (22) we are able to calculate the contribution of mobility fluctuations. For highly doped $\left(10^{20} \mathrm{~cm}^{-3}\right)$ silicon the diffusivity of holes is around $1 \mathrm{~cm}^{2} / \mathrm{s}$. The surface recombination velocity at the metal contact is in the order of $10^{5} \mathrm{~cm} / \mathrm{s}$. The hole recombination velocity at the oxide layer depends on its thickness. For a thickness of 7.5 $\AA-8 \AA$ we take [13] $s_{o x p} \approx 3 \cdot 10^{4} \mathrm{~cm} / \mathrm{s}$ and for $3.8 \AA$ we take $s_{\text {ox } p} \approx 8 \cdot 10^{4} \mathrm{~cm} / \mathrm{s}$. We use $W_{m} \approx W_{p} \approx 0.1 \mu \mathrm{m}$.

With $\alpha_{m}=\alpha_{p}=\alpha$, at $f=1 \mathrm{~Hz}$ we obtain $S_{I_{b}} \approx$ $3 \cdot 10^{-11} \alpha I_{B}$ for the total mobility fluctuation contribution. If we ascribe the deviations from the square law at low currents

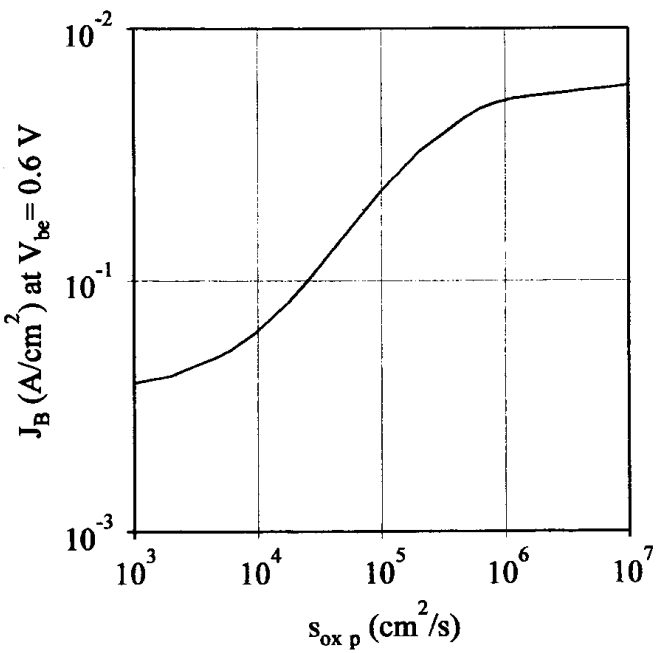

Fig. 7. Simulation of the base current density $J_{B}$ at $V_{b e}=0.6 \mathrm{~V}$ versus the oxide recombination velocity $s_{o x p}$.

in Fig. 3 to mobility fluctuations, we obtain $\alpha$ values in the range of $10^{-8}$ to $10^{-7}$. These values are quite low. With the values of $s_{o x p}$ used here, the conditions $s_{o x p} \gg W_{m} / \tau_{m}$, and $s_{\text {oxp }} \gg W_{p} / \operatorname{tau}_{p}$, that were used in the derivation of (17) and (21), are not entirely met. However, a numerical simulation of the base current density, $J_{B}$, as a function of the oxide recombination velocity $s_{o x p}$ using a reasonable doping profile, given in Fig. 7, proved that recombination of holes in the emitter becomes the dominant current contribution for $s_{\text {ox } p}<10^{4} \mathrm{~cm} / \mathrm{s}$. Hence, if no great accuracy is required, application of (17) and (21) is permissible for $s_{o x p}>10^{4} \mathrm{~cm} / \mathrm{s}$ and thus for oxide layers with a thickness of less than $13 \AA$ [13].

From (17) it is obvious that the oxide recombination velocity $s_{\text {oxp }}$ is the current limiting factor, provided that $s_{\text {oxp }} \ll$ $s_{m}, D_{m} / W_{m}, D_{p} / W_{p}$. In that case the influence of the mobility on the base current is weak. As a result, the $1 / f$ noise caused by fluctuations in the mobility, which is found in the base current, is reduced. The contribution of transparency fluctuations can be calculated with (18). Using the data previously mentioned, we find $S_{I_{b}} / I_{B}^{2} \approx 0.3 \cdot S_{s_{o x p}} / s_{o x p}^{2}=0.3 \cdot S_{t_{p}} / t_{p}^{2}$ for $7.5 \AA-8 \AA$ oxide layer thickness and $S_{I_{b}} / I_{B}^{2} \approx 0.1 \cdot S_{t_{p}} / t_{p}^{2}$ for $3.8 \AA$ thickness. With (10) we can calculate the loss factor $\tan \delta$ using $V_{0 h}=1.1 \mathrm{~V}$, and $m_{p}^{*}=0.42 m_{0}$, where $m_{0}$ is the electron rest mass. The capacitance of the oxide layer is given by

$$
C=\varepsilon A_{E} / L
$$

By applying this to the experimental data at high currents, presented in Fig. 5, we obtain values for $\tan \delta$. We find $\tan \delta$ to be 4 for the transistors with $3.8 \AA$ oxide layer, and 0.6 for transistors with $7.5 \AA$ and $8 \AA$ oxide layer. These values are rather high compared to the values reported in literature, that are of the order of $10^{-4}$ for quartz. However, the latter data are taken from bulk oxide material, whereas the oxide layers in our transistors only have thicknesses of a few atom layers. 
Moreover, the thickness and structure of the layer may not be completely uniform over the emitter area and the layer may even be interrupted at some spots, causing difficulty in the evaluation of (10).

In the derivation of (10) image force was neglected. However, the image force reduces the effective barrier height significantly for very thin oxide layers, causing an increase in the $1 / f$ noise. Thus, neglecting the image force leads to an overestimate of the $\tan \delta$.

In Section II.C we mentioned that the $1 / f$ noise, measured in the C-C configuration at high currents, was either ascribed to base resistance fluctuations or emitter resistance fluctuations. The contribution of emitter resistance fluctuations is claimed to be dominant for two reasons.

1) With only base resistance fluctuations we find the Hooge $1 / f$ noise parameter $\alpha$ to be in the range of $10^{-2}$ to $10^{-1}$, which is quite high.

2) $S_{V_{E}}$ depends on the oxide layer thickness at the polysilicon-monosilicon interface. $S_{r_{b}}$ is independent of the oxide thickness.

The emitter series resistance consists of three parts, a part located in the monosilicon $\left(r_{m}\right)$, in the oxide layer $\left(r_{o x}\right)$, and in the polysilicon layer $\left(r_{p}\right)$, thus

$$
r_{e}=r_{m}+r_{o x}+r_{p}
$$

The same holds for the $1 / f$ noise.

$$
S_{r_{e}}=S_{r_{m}}+S_{r_{o x}}+S_{r_{p}}
$$

The contribution of the $1 / f$ noise stemming from the monosilicon region was calculated using the Hooge relation [14].

$$
S_{r_{m}}=\alpha r_{m}^{2} / f N_{m}
$$

Here, $N_{m}$ is the total number of majority carriers in the monosilicon region.

De Graaff et al. [15] studied the $1 / f$ noise in polysilicon resistors with a dopant concentration in the range of $10^{15} \mathrm{~cm}^{-3}$ to $10^{20} \mathrm{~cm}^{-3}$. Their theoretical model has been improved by Luo et al. [16]. They derived the following relation for the $1 / f$ noise generated in the grain boundaries of the polysilicon.

$$
\frac{S_{I}}{I^{2}}=\frac{1}{N_{\mathrm{eff}}}\left(\frac{\nu_{r}}{\nu_{d}}\right)^{2} \frac{q d \alpha}{3 \varepsilon k T f A} \exp \left(\frac{q \Phi_{B}}{k T}\right) .
$$

Here, $N_{\text {eff }}$ is the effective number of large-barrier grains in the conduction path, $\nu_{r}$ the emission velocity over a grain boundary barrier, given by $\left(k T / 2 \pi m^{*}\right)^{1 / 2}, \nu_{d}$ the diffusion velocity, $d$ the depletion layer width, $\alpha$ the Hooge parameter, $A$ the the cross section of the polysilicon material, and $\Phi_{B}$ the grain boundary barrier height. For a dopant concentration of $10^{20} \mathrm{~cm}^{-3}$ De Graaff et al. [15] found a barrier height $\Phi_{B}$ of approximately $10 \mathrm{mV}$. They took $\nu_{d} \approx 2 \nu_{r}=1 / 4 \nu_{\text {thermal }}$. Applying (26) to the experiments of De Graaff et al. [15] yields an $\alpha$ value in the order of $10^{-5} \cdot\left(N / N_{\text {eff }}\right)$, with $N$ being the total number of grain boundaries in the conduction path. If we ascribe the measured noise in the emitter series resistance to

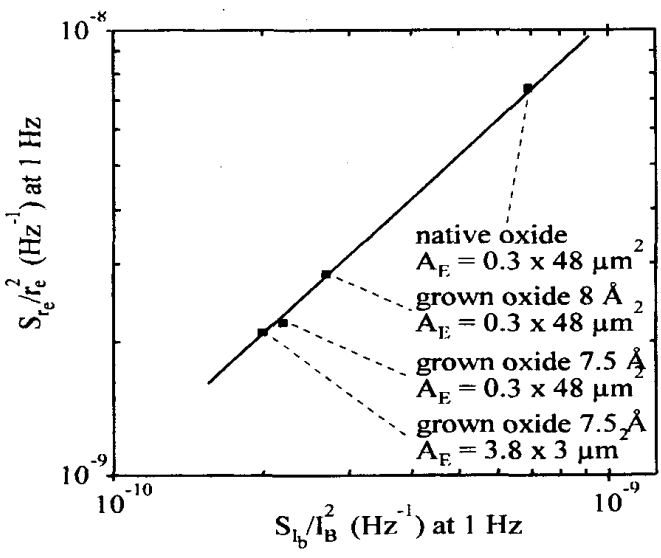

Fig. 8. $S_{I_{b}} / I_{B}^{2}$ versus $S_{r_{e}} / r_{e}^{2}$ for various oxide layer properties.

the polysilicon region and apply (26), we obtain $\alpha$ values in the order of $10^{-2} \cdot N_{\text {eff }}$. With a grain thickness of roughly $0.1 \mu \mathrm{m}$ we get a value for $N$ of about 5 , and $N_{\text {eff }} \leq 5$, which leads to a discrepancy between our results and those of [15].

If we ascribe the measured $1 / f$ noise to the monosilicon region only and apply (25), we obtain $\alpha$ values in the range $0.1 \leq \alpha \leq 1$, which are very high. In all probability the primary $1 / f$ noise source is located in the oxide layer.

In order to verify whether the measured $1 / f$ noise in the emitter series resistance can be ascribed to transparency fluctuations as predicted by our model (20), we plotted $S_{r_{e}} / r_{e}^{2}$ versus $S_{I_{b}} / I_{B}^{2}$ for transistors with various oxide layer properties. Both $S_{r_{e}} / r_{e}^{2}$ given by (20) and $S_{I_{b}} / I_{B}^{2}$ given by (18) are proportional to the relative noise in the transparency. As a result the plot of $S_{r_{\mathrm{e}}} / r_{e}^{2}$ versus $S_{I_{b}} / I_{B}^{2}$ has to be linear. The plot is given in Fig. 8. We observe that the plot is indeed linear.

A few remarks should be made.

1) $S_{r_{e}} / r_{e}^{2}$ is about factor 10 higher than $S_{I_{b}} / I_{B}^{2}$. This can be ascribed, first of all, to the prefactor $\left(d \ln I_{B} / d \ln s_{\text {ox } p}\right)^{2}$ in (18), previously calculated to be 0.3 for the transistors presented in Fig. 8. Secondly, the effective oxide barrier height for holes $(1.1 \mathrm{eV})$ is higher than for electrons $(0.6 \mathrm{eV})$. From (10) we conclude that $S_{t_{p}} / t_{p}^{2}$ is smaller than $S_{t_{n}} / t_{n}^{2}$.

2) We note that the factor $\left(d \ln I_{B} / d \ln s_{o x p}\right)^{2}$ in (18) depends on $s_{o x p}$. Consequently, the plot in Fig. 8 is only expected to be linear for transistors with nearly the same value of $s_{o x p}$, and thus for transistors with approximately the same oxide layer thickness. Since the emitter series resistance of the transistors presented in Fig. 8 have the same order of magnitude, it is assumed that all have about the same oxide layer thickness of nearby $8 \AA$.

3) Application of (20) is permissible provided that $r_{e}$ is determined by $r_{o x}$. Therefore, we have to certify that this condition is satisfied. In the monosilicon region $r_{m} A_{E}=\rho_{m} W_{m}$ is of the order of $10^{-1} \Omega \mu \mathrm{m}^{2}$. Here, $\rho_{m}$ is the resistivity of the monosilicon. The product $r_{p} A_{E}$ of the polysilicon region under low biasing 
conditions (i.e. $V_{g} \ll k T / q$ where $V_{g}$ is the voltage across a grain boundary) is given by [16]

$$
\frac{N_{\mathrm{eff}} k T}{q^{2} n(d) \nu_{r}} \exp \left(\frac{q \Phi_{B}}{k T}\right)
$$

Here, $n(d)$ denotes the electron denșity in the quasi-neutral regions.

By substituting the values mentioned previously, we obtain a value for $r_{p} A_{E}$ of the order of $10^{-1} \cdot N_{\text {eff }} \Omega \mu \mathrm{m}^{2}$.

Comparison of both $r_{m} A_{E}$ and $r_{p} A_{E}$ with the values measured in the range of $43 \Omega \mu \mathrm{m}^{2}-160 \Omega \mu \mathrm{m}^{2}$ reveals that $r_{e} \approx r_{o x}$. Hence, the emitter resistance is determined by the tunnel resistance and $S_{r_{o x}} / r_{o x}^{2} \approx S_{r_{e}} / r_{e}^{2}$.

\section{STRESS EXPERIMENTS}

Application of a sufficiently high reverse bias voltage to a emitter-base junction leads to an increase in the non-ideal base current. Generally, this also leads to an increase in the noise generated in the emitter-base junction. Thus, when we apply a reverse bias to the transistors studied here, we only expect the noise produced in the emitter-base junction to increase. The noise produced in the oxide layer at the monosiliconpolysilicon interface should not change since the stress is applied to the emitter-base junction only. In Fig. 9 the noise in the base current $S_{I_{b}}$ is plotted versus $I_{B}$, measured after different stress conditions. We observe that only the branch of the curves proportional to $I_{B}$ increases, whereas the branch of the curves proportional to $I_{B}^{2}$ is unchanged. These results confirm the existence of two different noise sources involved in the generation of $S_{I_{b}}$. The fact that application of stress does not affect the branch of $S_{I_{b}}$ proportional to $I_{B}^{2}$ agrees with our model.

\section{CONCLUSIONS}

The $1 / f$ noise of the polysilicon emitter bipolar transistors investigated in this study stems from noise in the base current $S_{I_{b}}$ and noise in the emitter series resistance $S_{r_{e}}$. By considering the $1 / f$ noise in $S_{I_{b}}$ as a function of the emitter geometry, we found that its $1 / f$ noise sources are homogeneously distributed over the emitter area. The $1 / f$ noise in $S_{I_{b}}$ and in $S_{r_{e}}$ increases with the thickness of the oxide layer present at the monosilicon-polysilicon interface. Grown oxide layers show less noise in both $S_{I_{b}}$ and $S_{r_{e}}$ than native oxide layers.

The $1 / f$ noise in $S_{I_{b}}$ can be explained in terms of transparency fluctuations, due to barrier height fluctuations of the oxide layer barrier at the polysilicon-monosilicon interface. These transparency fluctuations also cause the $1 / f$ noise in the emitter series resistance. Strong support for our model can be found in the fact that both $S_{I_{b}}$ and $S_{r_{e}}$ have the same dependence on the oxide layer properties.

Due to the low transparency of the oxide layer, the base current is almost independent of the diffusivity of minority carriers in the emitter. Thus the contribution of diffusivity (mobility) fluctuations to $S_{I_{b}}$ in the monosilicon region and the polysilicon region is reduced.

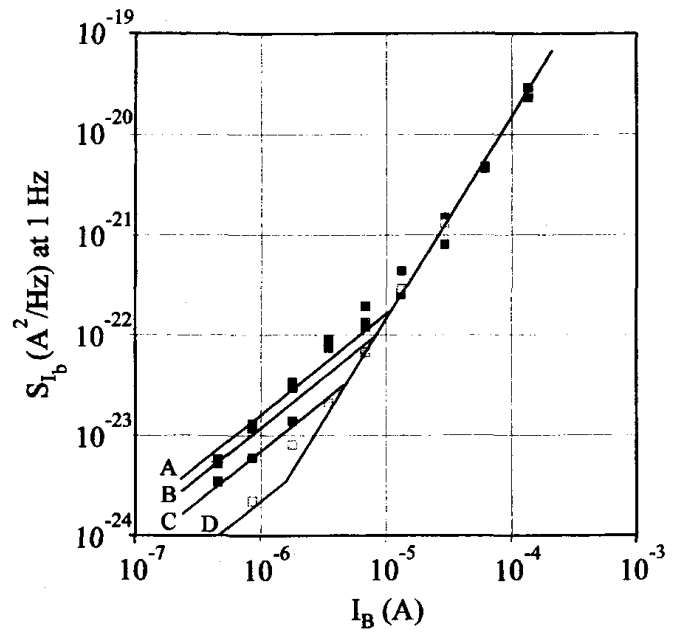

Fig. 9. $S_{I_{b}}$ versus $I_{B}$ for a transistor with a $7,5 \AA$ grown oxide layer and an emitter area of $100 \times 100 \mu \mathrm{m}^{2}$ measured without stress (D), and after 30 min. stress at $4 \mathrm{~V}(\mathrm{C}), 6 \mathrm{~V}(\mathrm{~B})$, and $7 \mathrm{~V}$ (A).

\section{ACKNOWLEDGMENT}

The transistors were manufactured by Philips Research Laboratories. The authors would like to thank Dr. G. A. M. Hurkx and J. de Boet, staff members of Philips, for providing the transistors and for their close cooperation.

\section{APPENDIX \\ RELATION FOR THE CONTRIBUTION OF MOBILITY FluCtuATIONS TO $S_{I_{B}}$}

By means of (11)-(15) the current fluctuations around the steady-state due to diffusivity fluctuations are found to be

$$
\begin{aligned}
\Delta I(x, t) & =\frac{I}{D_{m, p}} \Delta D_{m, p}(x, t)-q A D_{m, p} \frac{d \Delta p(x, t)}{d t} \\
\Delta I\left(x_{3}, t\right) & =q A s_{m} \Delta p\left(x_{3}, t\right)
\end{aligned}
$$

and

$$
\begin{aligned}
\Delta I\left(x_{1}, t\right) & =\Delta I\left(x_{2}, t\right) \\
& =q A s_{o x p}\left[\Delta p\left(x_{1}, t\right)-\Delta p\left(x_{2}, t\right)\right] .
\end{aligned}
$$

Integrating (A1) over the monosilicon and polysilicon region yields

$$
\Delta I(t)=\frac{1}{W_{m}} \int_{0}^{x_{1}} \Delta I(x, t) d x=\frac{1}{W_{p}} \int_{x_{2}}^{x_{3}} \Delta I(x, t) d x
$$

and thus

$$
\begin{aligned}
\Delta I(t)= & \frac{I}{W_{m}} \int_{0}^{x_{1}} \frac{\Delta D_{m}(x, t)}{D_{m}} d x-q A \frac{D_{m}}{W_{m}} \Delta p\left(x_{1}, t\right) \\
= & \frac{I}{W_{p}} \int_{x_{2}}^{x_{3}} \frac{\Delta D_{p}(x, t)}{D_{p}} d x-q A \frac{D_{p}}{W_{p}} \\
& \cdot\left[\Delta p\left(x_{3}, t\right)-\Delta p\left(x_{2}, t\right)\right] .
\end{aligned}
$$

If the junction is short circuited, $p(0)$ does not fluctuate, thus $\Delta p(0, t)=0$. With the help of (A2) the fluctuation 
$\Delta p\left(x_{3}, t\right)$ in (A5) can be expressed in terms of $\Delta I(t)$. With (A3) the fluctuation $\Delta p\left(x_{2}, t\right)$ in (A5) can be expressed in $\Delta p\left(x_{1}, t\right)$. Now (A5) consists of two equations with two unknown parameters: $\Delta p\left(x_{1}, t\right)$ and $\Delta I(t)$. For $\Delta I(t)$ we obtain

$$
\Delta I(t)=\frac{\frac{I}{D_{m}} \int_{0}^{x_{1}} \frac{\Delta D_{m}(x, t)}{D_{m}} d x+\frac{I}{D_{p}} \int_{x_{2}}^{x_{3}} \frac{\Delta D_{p}(x, t)}{D_{p}} d x}{\frac{W_{m}}{D_{m}}+\frac{W_{p}}{D_{p}}+\frac{1}{s_{\text {oxp }}}+\frac{1}{s_{m}}} .
$$

By making a Fourier transform, and using the crosscorrelation spectral noise density in the diffusivity, given by [14]

$$
S_{D}\left(x, x^{\prime}, f\right)=\frac{\alpha D^{2}}{f A p(x)} \delta\left(x-x^{\prime}\right)
$$

with $\alpha$ the Hooge parameter, and $\delta$ the Dirac delta function, we obtain (21).

\section{REFERENCES}

[1] T. G. M. Kleinpenning, "Location of low-frequency noise sources in submicrometer bipolar transistors," IEEE Trans. Electron Devices, vol. 39 , pp. 1501-1506, 1992.

[2] P.-F. Lu, "Low-frequency noise in self-aligned bipolar transistors," $J$. App. Phys., vol. 62, pp. 1335-1339, 1987.

[3] A. H. Pawlikiewicz, A. van der Ziel, G. S. Kousik, and C. M. van Vliet, "Fundamental $1 / f$ noise in silicon bipolar transistors," Solid-State Electron., vol. 31, pp. 831-834, 1988.

[4] N. Siabi-Shahrivar, H. A. Kemhadjian, W. Redman-White, P. Ashburn, and J. D. Williams, "The effects of scaling and rapid thermal annealing on the $1 / f$ noise of polysilicon emitter bipolar transistors," Microelectronic Engineering, vol. 15, pp. 533-536, 1991.

[5] A. Mounib, F. Balestra, N. Mathieu, J. Brini, G. Ghibaudo, and A Chovet, "Low-frequency noise sources in polysilicon emitter bipolar transistors: Influence of hot-electron-induced degradation," in Noise in Physical Systems and $1 / f$ Fluctuations, P. H. Handel and A. L. Chung, Eds., AIP Conference Proceedings 285. New York: American Institute of Physics, 1993, pp. 288-291.

[6] W. S. Lau, E. F. Chor, C. S. Foo, and W. C. Khoong, "Strong lowfrequency noise in polysilicon bipolar transistors with interfacial oxide due to fluctuations in tunneling probabilities," Jpn. I. Appl. Phys., vol. 31, pp. L1021-L1023, 1992.

[7] H. Higuchi and S. Ochi, "1/f Noise characteristics of bipolar transistors," Proc. Symp. on 1/f Fluctuations, Tokyo, p. 140, 1977.
[8] T. G. M. Kleinpenning, " $1 / f$ Mobility fluctuations in $\mathrm{p}-\mathrm{i}-\mathrm{n}$ and $\mathrm{p}-\nu-\mathrm{n}$ diodes," Physica B vol. 154, pp. 27-34, 1988.

[9] "On low frequency noise in tunnel diodes," Solid-State Electr., vol. 21 , pp. 927-931, 1978.

[10] vol. 25 , pp. 79-80, 1982.

[11] Z. Yu, B. Riccó and R. W. Dutton, "A comprehensive anaytical and numerical model of polysilicon emitter contacts in bipolar transistors," IEEE Trans. Electron Devices, vol. ED-31, pp. 773-784, 1984.

[12] V. Kumar and W. E. Dahlke, "Low-frequency noise in $\mathrm{Cr}-\mathrm{SiO}_{2}-\mathrm{n}-\mathrm{Si}$ tunnel diodes," IEEE Trans. Electron Devices, vol. 24, pp. 146-153, 1977.

[13] S. M. Sze, "High-speed semiconductor devices," in Interscience. New York: Wiley, ISBN 0-471-62307-5, 1990.

[14] F. N. Hooge, T. G. M. Kleinpenning, and L.K.J. Vandamme, "Experimental studies on $1 / f$ noise," Rep. Prog. Phys., vol. 44, pp. 479-532, 1981.

[15] H. C. de Graaff and M. T. M. Huybers, " $1 / f$ noise in polycrystalline silicon resistors," J. Appl. Phys., vol. 54, no. 5, pp. 2504-2507, 1983.

[16] M.Y. Luo and G. Bosman, "An analytical model for $1 / f$ noise in polycrystalline silicon thin films," IEEE Trans. Elelctron Devices, vol. 37, pp. 768-774, 1990.

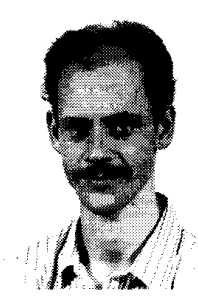

Hans A. W. Markus was born in Beuningen, The Netherlands, on December 8, 1968.

$\mathrm{He}$ is currently working towards the Ph.D. degree in electrical engineering at the Eindhoven University of Technology, Eindhoven, The Netherlands. His research concerns the electrical noise in semiconductor devices.

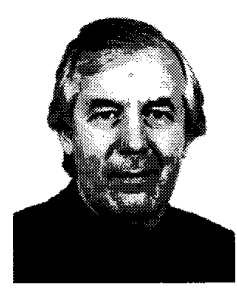

Theo G. M. Kleinpenning was born in Doetinchem, The Netherlands, on May 25, 1943. He received the $\mathrm{Ph} . \mathrm{D}$. degree in physics from the State University of Utrecht, The Netherlands, in 1972.

In 1972, he joined the Department of Electrical Engineering of the Eindhoven University of Technology, Eindhoven, The Netherlands, where he is currently a Full Professor of Semiconductor Devices. His current research concerns the electrical noise in semiconductors and semiconductor devices. He has published over 60 scientific papers on noise. 